\title{
Intraoperative Musculoskeletal Injury, CTCAE
}

National Cancer Institute

\section{Source}

National Cancer Institute. Intraoperative Musculoskeletal Injury, CT CAE. NCI Thesaurus.

Code C143606.

A finding of damage to the musculoskeletal system during a surgical procedure. 